\title{
IQGAP1 promotes cell proliferation and is involved in a phosphorylation-dependent manner in wound closure of bronchial epithelial cells
}

\author{
YONGPING WANG, MANXIANG WANG, FANG WANG, \\ MIN ZHU, YAN MA, XI WANG and RENLIANG WU \\ Institute of Pathology, Tongji Hospital, Tongji Medical College, Huazhong University of Science and \\ Technology, Key Laboratory of Pulmonary Disease of Ministry of Health of China, Wuhan 430030, P.R. China
}

Received March 3, 2008; Accepted April 24, 2008

\begin{abstract}
The re-epithelialization process of the airway involves spreading and migration followed by cell proliferation. Scaffold IQ domain GTPase-activating protein (IQGAP1), an effector of Rho GTPases, is a key component in a series of cell processes, although its exact mechanism in injury and repair of the airway is still unclear. In this study, we utilized a widely used model in vitro by scratching bronchial epithelial cells (BECs). At different time points after scratching, the amounts of IQGAP1 in mRNA and protein were greater than that in the control. PKCE-mediated phosphorylation of IQGAP1 was involved in the process of injury and repair. The overexpression of $\mathrm{PKC} \varepsilon$ or treatment with phorbol-12-myristate-13-acetate (the PKC activator) promoted wound closure. On the contrary, the group treated with GF109203X (the PKC inhibitor) had the opposite effect.
\end{abstract}

Correspondence to: Professor Renliang Wu, Institute of Pathology, Tongji Hospital, Tongji Medical College, Huazhong University of Science and Technology, Key Laboratory of Pulmonary Disease of Ministry of Health of China, No.13 Hangkong Road, Wuhan 430030, Hubei, P.R. China

E-mail: renliangwu@hotmail.com

Abbreviations: IQGAP1, IQ domain GTPase-activating protein; BECs, bronchial epithelial cells; MAPs, microtubule-associated proteins; Tcf/Lef, T cell factor/lymphoid enhanced factor; COPD, chronic obstructive pulmonary disease; CLIP-170, cytoplasm linking protein 170; APC, adenomatous polyposis coli; LIS1, the product of the Lissencephaly 1 gene; PIC, protease inhibitor cocktail; PMA, Phorbol-12-myristate-13-acetate; ECL, enhanced chemiluminescence; PMSF, p-amidinophenyl-methanesulfonyl fluoride; HEPES, 4-(2-hydroxyerhyl) piperazine-1-erhanesulfonic acid; FBS, fetal bovine serum; qRT-PCR, quantitative reverse transcriptase-polymerase chain reaction; CT, threshold cycle; GAPDH, glyceraldehyde-3-phosphate-dehydrogenase; SDS-PAGE, SDS polyacrylamide gel electrophoresis; GFP/EGFP, green fluorescence protein/enhanced green fluorescence protein; siIQGAP1, small interference RNA of IQGAP1

Key words: IQ domain GTPase-activating protein, phosphorylation, cell proliferation, injury and repair, bronchial epithelial cells
Scratching or overexpression of IQGAP1 induced increasing amounts of total $B$-catenin and the transposition of $B$-catenin from the cytoplasm into the nucleus. These results activated the $\mathrm{T}$ cell factor/lymphoid enhanced factor and induced expression levels of its target genes of c-myc and cyclin D1. The reduction of IQGAP1 by the transfection of small interference RNA of IQGAP1 attenuated these effects and directly impaired the scratching-induced wound closure. Taken together, our results suggest that IQGAP1 promotes cell proliferation and phosphorylation of IQGAP1 is involved in the process of wound closure in BECs.

\section{Introduction}

The airway (trachea and bronchi) is frequently injured because of its exposure to the external environment. After injury, the airway epithelium undergoes a wound repair process, which initiates spreading, migration and proliferation to overlay the injured area (1). In the process of wound repair of the airway epithelium, the cells around the wound initially become flat, then disseminate and migrate toward the wound area. Eventually, basal cells proliferate and differentiate into ciliated epithelium with the recovery of the epithelium $(2,3)$. These actions depend on the complicated functions of the Rho family GTPases and the cytoskeleton (4). It is an important step that the airway epithelium initiates the wound repair process after injury in the resolution of airway diseases such as chronic bronchitis and chronic obstructive pulmonary disease (COPD), which are characterized by chronic injury and inefficient repair.

As an important effector of Rho GTPases, IQ domain GTPase-activating protein (IQGAP1) is an integral protein of cytoskeletal organization and interacts with many targets such as actin (5), ß-catenin (6), E-cadherin (7), CLIP-170 (8) (cytoplasm linking protein), APC (9) (adenomatous polyposis coli) and LIS1 (the product of the Lissencephaly 1 gene) (10). These targets are important components of cell adhesion and microtubule-associated proteins (MAPs), thus IQGAP1 is considered to play a role in cell adhesion, polarization and migration $(11,12)$.

Phosphorylation is a major post-translational method for regulating protein function, including that of the cytoskeleton. More evidence reveals that phosphorylation is essential for 
Ena/VASP function in cell movement (13) and the activity of Rho is regulated by tyrosine phosphorylation of RhoGEFs (14). The serine residues of IQGAP1 are phosphorylated and PKC $\varepsilon$ may be one of the candidates responsible for this effect. This result promotes neurite outgrowth $(15,16)$.

We have found that the $\beta$-catenin/Tcf signal is involved in airway squamous metaplasia, APC and CLIP-170 are closely controlled both spatially and temporally in injury and repair of bronchial epithelial cells (BECs) (17-20). Since IQGAP1 is associated with targets of MAPs and cell adhesion and MAPs are regulated by Rho GTPases, it is significant to know whether and how IQGAP1 (phosphorylated IQGAP1) works in the process of injury and repair. In support of this hypothesis, we utilized a model of injury and repair of BECs in vitro, which was available for cell injury and migration (21). Our results give new insight into IQGAP1 in injury and repair and these findings may be helpful in understanding the mechanism of the process.

\section{Materials and methods}

Reagents. Penicillin, streptomycin and HEPES were obtained from Sigma Chemical (St. Louis, MO, USA). DMEM/F-12 and FBS were from Gibco Company (Carlsbad, CA, USA). The protease inhibitor cocktail (PIC) was obtained from Calbiochem (St. Louis, MO, CA, USA). NE-PER ${ }^{\mathrm{TM}}$ nuclear and cytoplasmic extraction regents, the BCA kit and enhanced chemiluminescence (ECL) were purchased from Pierce Chemical Company (Rockford, IL, USA). The antibodies, including anti-PKC $\varepsilon$, anti- $\beta$-catenin, antiGAPDH, anti-GFP, anti-c-myc, anti-cyclin D1, anti-tubulin, anti-lamin $\mathrm{B}$ and protein A-agarose beads were the products of Santa Cruz Biotechnology, Inc (Santa Cruz, CA, USA). Anti-ß-actin, anti-IQGAP1 and anti-phosphorylated-serine antibodies were from MBL (Woburn, MA, USA), Zymed (South San Francisco, CA, USA) and Chemicon International (Temecula, CA, USA), respectively. The PKC activator PMA and inhibitor GF109203X were obtained from BioSource International Inc. (BioSource, Camarillo, CA). Lipofectamine 2000, TriZol and primers of IQGAP1, B-actin and GAPDH were from Invitrogen Life Technology (Invitrogen, CA, USA). The luciferase assay kit and the $\beta$-galactosidase assay kit were the products of Promega Corporation (Promega, Madison, USA). Materials used in the nucleic acid study were purchased from Takara Shuzo Co., Ltd (Takara, Kyoto, Japan). Other materials and chemicals were obtained from commercial sources.

Cell culture, injury and repair model and wound measure of BECs. 16HBE140- cells, a simian virus 40 large T antigentransformed human bronchial epithelial cell line that maintains tight junctions (22) was generously provided by Dr D. Gruenert (California Pacific Medical Center, CA, USA). 16HBE14o- cells were cultured in DMEM supplemented with $10 \%$ FBS and $1 \%$ penicillin-streptomycin solution buffered in $20 \mathrm{mM}$ HEPES and $2.2 \mathrm{~g} / \mathrm{NaHCO}_{3}, \mathrm{pH} 7.2$, at $37^{\circ} \mathrm{C}$ and $5 \%$ $\mathrm{CO}_{2}$. Each of the experiments were performed and repeated in one set of 16HBE140- cells.

An injury and repair model of the airway epithelium in vitro was utilized by scratching on the cultured BECs as described previously (19). Briefly, BECs were cultured in a complete medium in $90-\mathrm{mm}$ diameter dishes or $6 / 12$-well plates. When cells were $80 \%$ confluent, the medium was changed to the serum-free counterpart. After being maintained for $6 \mathrm{~h}$, the cells were scratched. The multiple wounds were produced by scratching the cell horizontally and vertically with an 8-channel pipette (with $10 \mu 1$ tip) across the well. Then, the cells were harvested at different time points for further analysis.

The mean wound width was calculated using a standard cell culture microscope equipped with an ocular micrometer. Data were expressed as a percentage of $0 \mathrm{~h}$ wound width to normalize variability in wounding from experiment to experiment. All the results reported were from six independent wells from two separate experiments. Images were obtained at the initial time of wounding and then at various times.

Reverse transcription-polymerase chain reaction (RT-PCR) and quantitative RT-PCR. Briefly, total RNA was harvested from BECs using TriZol $\left(1 \mathrm{ml} / 10 \mathrm{~cm}^{2}\right)$, then isolated and precipitated by chloroform and isoamyl alcohol. RNA was washed with $75 \%$ alcohol and dried for $10 \mathrm{~min}$ at room temperature, then dissolved in pure water without RNase. The RNA quality was assessed by agarose gel electrophoresis and was quantitated spectrophotometrically. Reverse transcription was performed using $4 \mu \mathrm{g}$ of RNA, $0.5 \mu \mathrm{g}$ oligo dT and DEPC $\mathrm{H}_{2} \mathrm{O}$ in a total volume of $12 \mu \mathrm{l}$ system. At $70^{\circ} \mathrm{C}$ after $5 \mathrm{~min}$, the mixture was cooled on ice for $1 \mathrm{~min}$ and was then added with $5 \mathrm{X}$ buffer, RNAsin, RT-dNTP, reverse transcriptase. The cDNA was harvested after the mixture was treated by the following steps: $42^{\circ} \mathrm{C}$ for $1 \mathrm{~h}, 70^{\circ} \mathrm{C}$ for $10 \mathrm{~min}$, cooling on ice and $95^{\circ} \mathrm{C}$ for $5 \mathrm{~min}$. The PCR reaction was carried out in a total volume of $25 \mu \mathrm{l}$ containing $15 \mu 1 \mathrm{H}_{3} \mathrm{O}, 2.5 \mu \mathrm{l}$ 10X buffer, $2 \mu 1 \mathrm{MgCl}_{2}, 0.5 \mu \mathrm{ldNTP}, 0.5 \mu 1$ primers, $2 \mu \mathrm{l}$ cDNA, $2 \mu 1$ Taq enzyme and $10 \mu 1$ mineral oil. Amplification was performed using a PCR instrument (MJ Research, Inc., USA) at $94^{\circ} \mathrm{C}$ for $5 \mathrm{~min}$, followed by 45 cycles at $94^{\circ} \mathrm{C}$ for $30 \mathrm{sec}, 58^{\circ} \mathrm{C}$ for $40 \mathrm{sec}$ and $72^{\circ} \mathrm{C}$ for $30 \mathrm{sec}$. PCR products were checked by electrophoresis on $2 \%$ agarose gels, based on the size of the amplified cDNA fragments.

Quantitative PCR was carried out using the SYBR-Green PCR kit (Takara, Osaka, Japan) in a Rotor-Gene 3000 machine (Corbett Life Science, Sydney, Australia). Quantitative analysis of IQGAP1 transcription was applied by the comparative threshold cycle (CT) method for the calculation of amplification fold as specified by the manufacturer. Commercially available primers for GAPDH mRNA were used for normalization (Invitrogen Life Technology) and gene-specific IQGAP1 primers were designed with primer premier 6.0 software. Results were normalized against GAPDH and expressed in relation to a calibrator sample. Results per PCR reaction were expressed as a relative gene expression, using the $\delta$ - $\delta$ CT method (23) $(\delta-\delta$ CT is the differences of CT between the target products and the control). The calibrator was chosen among the control and was given a relative expression value of 1 . The reactions were carried out with the following reagents and mixed gently: $15 \mu 1$ of SYBR-Green Mastermix, $0.5 \mu \mathrm{l}$ of $10 \mu \mathrm{M}$ forward/reverse primer, $2 \mu 1$ of template and $7 \mu 1$ nuclease-free water to a total volume of $25 \mu \mathrm{l}$. The following were the sequences of 
primers: IQGAP1: For: 5'-CACTGGCTAAGACGGAAGT GTC-3', Rev: 5'-TCCGGTGCGGAT-3', product of $140 \mathrm{bp}$. GAPDH: For: 5'-ATGACATCAAGAAGGGGTG-3', Rev: 5'-CTACCAGGAAATGAGCTTG-3', product of $177 \mathrm{bp}$.

Immunoprecipitation and Western blot analysis. The immunoprecipitation assay was performed as described by us (19). In brief, treated BECs were harvested and washed with ice-cold PBS containing $1 \mathrm{mM}$ orthovanadate, then lysed at $4^{\circ} \mathrm{C}$ in Nonidet $\mathrm{P}-40$ buffer $[10 \mathrm{mM}$ Tris/ $\mathrm{HCl}(\mathrm{pH} 7.5)$, $140 \mathrm{mM} \mathrm{NaCl}, 1 \mathrm{mM}$ orthovanadate, $1 \%$ Nonidet P-40, $2 \mathrm{mM}$ PMSF, $10 \mathrm{mM}$ EDTA, $2 \mu \mathrm{g} / \mathrm{ml}$ PIC]. Nuclei were discarded following centrifugation at $12,000 \mathrm{x} \mathrm{g}$ for $15 \mathrm{~min}$. Lysates were incubated for $1 \mathrm{~h}$ at $4{ }^{\circ} \mathrm{C}$ with protein A-agarose to precipitate the non-specificity proteins. The supernatants were mixed with specific antibodies and incubated overnight at $4^{\circ} \mathrm{C}$ with gentle agitation, then incubated with protein A-agarose for $3 \mathrm{~h}$ at $4^{\circ} \mathrm{C}$. Precipitates were collected by centrifugation and extensively washed in Nonidet P-40 buffer. The same amounts of proteins ( $5 \mu \mathrm{g} / \mathrm{lane})$ were loaded in different lanes, and analyzed by SDS-PAGE. The antibodies and dilutions included anti-PKCE (1:400), antiIQGAP1 (1:500), anti-GFP (1:500) and anti-phosphorylated serine (1:400) antibodies.

In Western blot analysis, treated cells were rinsed twice with ice-cold PBS, collected by trypsinization $(0.1 \%$ trypsin $+0.02 \%$ EDTA $)$ and lysed in buffer $(50 \mathrm{mmol} / 1$ Tris-HCl, pH 8.0, $100 \mathrm{mmol} / \mathrm{l} \mathrm{NaCl}, 1 \mathrm{mmol} / \mathrm{l}$ EDTA, 1 mmol/l dithiothreitol, $1 \%$ Triton X-100, 0.1\% SDS, 50 mmol/1 sodium fluoride, $1 \mathrm{mmol} / 1$ sodium vanadate and $1 \%$ aprotease inhibitor cocktail). In order to get cytoplasmic and nuclear extracts, cells were lysed with NE-PER nuclear and cytoplasmic extraction reagents plus protease inhibitors according to the instructions from the manufacturer. Cell lyasates were cleared by centrifugation and protein concentration was determined by a BCA kit. Equal amounts of proteins were fractionated by SDS-polyacrylamide gel electrophoresis and transferred onto a nitrocellulose membrane. The membranes were blocked with $5 \%$ non-fat milk in TBST and incubated with anti-IQGAP1 (1:400), anti-GAPDH (1:500), anti-tubulin (1:500), anti-lamin B (1:500), anti-ß-catenin (1:400), anti-cmyc (1:400), anti-cyclin D1 (1:400) and anti- $\beta$-actin (1:500) antibodies overnight at $4{ }^{\circ} \mathrm{C}$. The signal was detected by using a horseradish peroxidase-conjugated secondary antibody and enhanced chemiluminescence (ECL) and then exposed to X-ray film (Fuji, Japan).

RNA interference. A pool of three RNA oligomers containing 21 nucleotides of human IQGAP1 were synthesized (Santa Cruz Biotechnology) in sense and antisense directions corresponding to nucleotides 2093-2111, 2404-2422 and 2513-2531(NM_003870.3), respectively. The sequences with dTdT overhangs at each 3 termini were as follows: A: sense strand 5'-CCACAGUGAUCUUGCUGAA-3'; B: sense strand 5'-GGAUGAAUUUCCUGAAGAA-3'; C: sense strand 5'-CCACAAAGAUGAAGUUGUA-3'. Unspecific negative (scramble) and positive ( $\beta$-actin) controls were used. Transfection was carried out using Lipofectamine 2000 reagent and the interference effects were evaluated by RT-PCR and Western blotting.
Plasmids and transient transfection. The plasmid of pEGFP-IQGAP1 (WT) was generously provided by Professor Kozo Kaibuchi (Department of Cell Pharmacology, Nagoya University Graduate School of Medicine, Nagoya, Japan) as described (24). The pcDNA-PKCE plasmid was a generous gift from Professor Cao Xi'nan (Laboratory of molecular biology, Kunming Medical College, Kunming, P.R. China). Tcf luciferase reporter plasmids (pGL3-OT and pGL3-OF, improved versions of pTOPFLASH and pFOPFLASH) (25) were generous gifts from Dr Bert Vogelstein (The Sidney Kimmel Comprehensive Cancer Center, the Johns Hopkins Medical Institutions, USA). Each construct harbors an Xho fragment containing three copies of wild-type (CCTTTGATC, pGL3-OT) or mutant (CCTTTGGCC, pGL3-OF) human Tef-4 binding site cloned into the pGL3-Basic plasmid. Transient transfection of the plasmids described above was carried out using Lipofectamine 2000 according to the recommendation from the manufacturer and a method (26) with minor modification.

Luciferase reporter assay. When confirmation of $70-80 \%$ confluence, BECs were co-transfected with Tcf luciferase reporter plasmids $(0.5 \mu \mathrm{g}$ pGL3-OT, -OF) and IQGAP1 plasmid (0.5 $\mu \mathrm{g}$ pEGFP-IQGAP1). Meanwhile, cells in each group were co-transfected with a ß-galactosidase expression vector $(0.1 \mu \mathrm{g} \mathrm{pCH} 110)$ for normalizing the transfection efficiency. After transfection for $24 \mathrm{~h}$, cells detected the activities of luciferase and $\beta$-galactosidase using commercial kits as directed by the manufacturer's instructions. Luciferase activity was measured with Lumat LB9507 luminometer (EG\&G Berthold, Germany) and normalized for B-galactosidase activity (OD420).

Statistical analysis. Statistical analysis was performed with the SPSS statistical software (SPSS 12.0). One-way ANOVA was performed for comparisons of multiple treatments (Fisher's LSD) and the Dunnet test was used for comparing other groups against the control group. A Student's t-test was then performed in order to determine significant differences between individual conditions. Significant differences were determined based on a threshold of $\mathrm{P}<0.05$.

\section{Results}

Scratching induced expression levels of IQGAPI in $m R N A$ and protein. The cultured BECs displayed a classic cobblestone-like epithelial morphology under a phase contrast microscope. After scratching, the anterior border cells moved towards the wound as sheets or groups, perpendicular in the direction of the gap. Six hours later, the frontal cells showed a polarized morphology. We observed cell migrations or proliferations at $12 \mathrm{~h}$ after injury. The wound closure occurred $24 \mathrm{~h}$ after scratching (Fig. 1A).

A notable finding revealed that IQGAP1 activates the transcription of $\beta$-catenin (27). Considering the process of wound closure (Fig. 1A) and a previous report (21), we selected $0,0.5,1,2,3,6,9,12$ and $24 \mathrm{~h}$ as time points for scratching. The amounts of IQGAP1 in mRNA increased and were at a maximum at $6 \mathrm{~h}$ after scratching BECs (Fig. 1B and D) ( ${ }^{*} \mathrm{P}<0.05$ compared with $\left.0 \mathrm{~h}\right)$. The process lasted for $\sim 24 \mathrm{~h}$. 


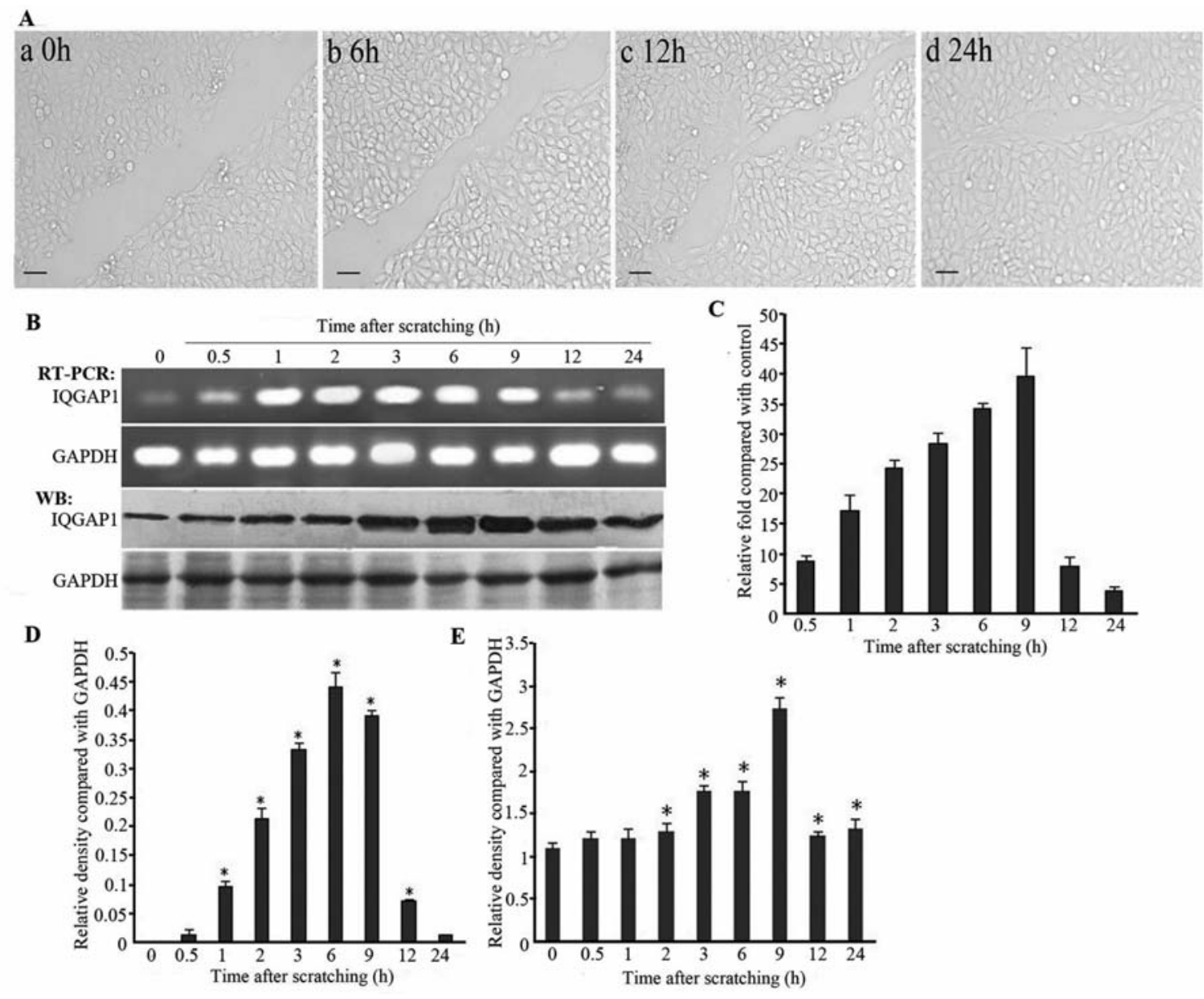

Figure 1. Scratching increased the amounts of IQGAP1 in mRNA and protein. (A) BECs were visualized under a phase contrast microscope after being scratched and showed a classic cobblestone epithelial morphology with closely adherent (a). After being scratched for $6 \mathrm{~h}$ or $12 \mathrm{~h}$, the cells moved towards the wound and were perpendicular to the wound direction (b and c). The closure of the wound occurred $\sim 24 \mathrm{~h}$ after scratching (d). Scale bar, $50 \mu \mathrm{m}$. (B) At different time points after scratching, total mRNA of IQGAP1 was collected. RT-PCR was carried out and the products were assessed by $2 \%$ agarose gel electrophoresis. Then, BECs were lysed and immunoblotted (WB) with GAPDH and IQGAP1 antibodies at the same time points. Data (D, mRNA; E, protein) represent the means of the independent experiments which are repeated three times $\left({ }^{*} \mathrm{P}<0.05\right.$ compared with $0 \mathrm{~h}$ ). (C) Fluorescent quantitative PCR was performed with SYBR-Green Mastermix addition to the PCR system. After comparing the amplifying efficiency with GAPDH, the amplification data were analyzed with the $\delta-\delta$ CT method. The calibrator was chosen among the control and was given a relative expression value of 1 . The black column represents the relative amplification folds of IQGAP1 contrast to GAPDH. Data represent the means from three independent experiments.

Then, we used qRT-PCR for an exact examination of IQGAP1 in mRNA. A comparative $\delta-\delta C T$ method was applied to show differences of CT between IQGAP1 and control (see Materials and methods). Similar to the above result, the amounts of IQGAP1 in mRNA increased and got the maximum at $9 \mathrm{~h}$, which were $\sim 40$-fold to $0 \mathrm{~h}$ (Fig. 1C). The amounts of IQGAP1 in protein also increased after scratching (Fig. 1B and E) $\left({ }^{*} \mathrm{P}<0.05\right.$ compared with $\left.0 \mathrm{~h}\right)$.

$P K C$ - - mediated phosphorylation of IQGAP1 was involved in the process of wound closure induced by scratching. IQGAP1 is highly phosphorylated at multiple serine sites after stimulation, which affects its function of regulating the cytoskeleton (16). To detect phosphorylation of IQGAP1 in BECs, we pulled down precipitates and detected it with anti-IQGAP1 and anti-phosphorylated serine antibodies, respectively. Soon after scratching, the phosphorylation of IQGAP1 increased and reached a maximum at $1 \mathrm{~h}$, lasting for at least $9 \mathrm{~h}$ (Fig. 2A and C).

There are three classes of protein kinase $\mathrm{C}$ isoforms, conventional, novel and atypical. $\mathrm{PKC} \varepsilon$ is a member of novel
PKC and is reported as a candidate for the phosphorylation of IQGAP1 $(15,16)$. We tried to identify the relationship between $\mathrm{PKC} \varepsilon$ and the phosphorylation of IQGAP1 in BECs. Overexpression of PKC $\varepsilon$ or treatment with PMA (the PKC activator, $0.1 \mu \mathrm{M})$ for $4 \mathrm{~h}$ increased the amounts of phosphorylation of IQGAP1 (Fig. 2B and D) $\left({ }^{*} \mathrm{P}<0.05\right.$ compared with the control before scratching). Considering the results in Fig. 2A, we selected $1 \mathrm{~h}$ as the time point for scratching. In groups for scratching, cells were pre-treated (or not) with GF109203X (the PKC inhibitor, $20 \mu \mathrm{M}$ ) for $3 \mathrm{~h}$, then scratched and incubated for $1 \mathrm{~h}$. We found that phosphorylation of IQGAP1 induced by scratching was blocked by GF109203X, which was opposite to the control. We then studied the relationship between IQGAP1 and PKC $\varepsilon$ by immunoprecipitation in BECs. IQGAP1 and PKC $\varepsilon$ were coimmunoprecipitated with each other (Fig. 2E). From the figure, we found that scratching induced a close association between IQGAP1 and PKCE (data not shown).

Our further steps focused on the direct effects of phosphorylation of IQGAP1 in wound closure. After treatment with PMA $(0.1 \mu \mathrm{M})$, GF109203X $(20 \mu \mathrm{M})$ or overexpression 


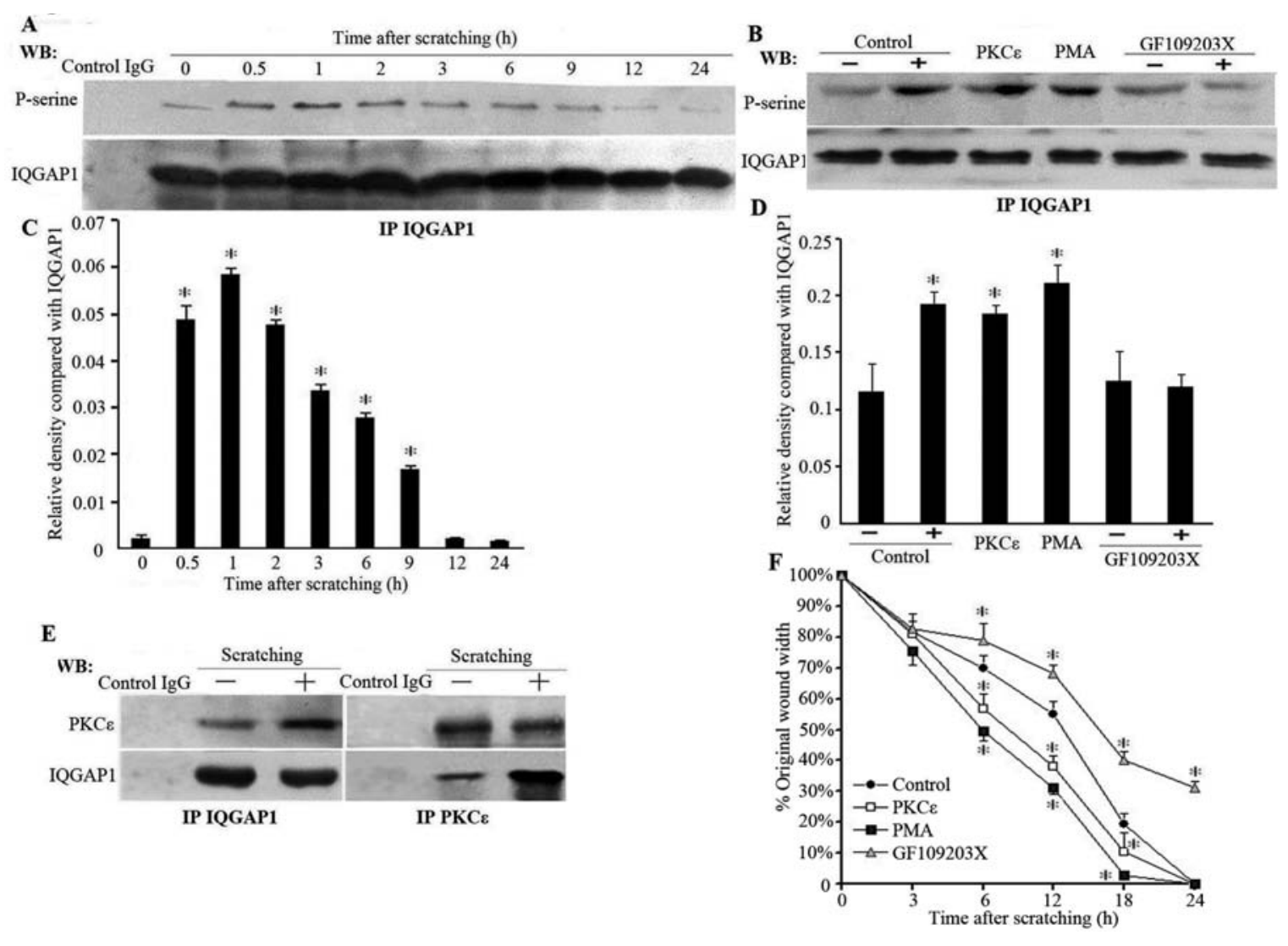

Figure 2. PKCE-mediated phosphorylation of IQGAP1 was involved in the process of wound closure. (A) After being scratched, BECs were lysed and immunoprecipitated (IP) with IQGAP1. Phosphorylated-serine was detected in the equal amounts of precipitates by Western blotting (WB). Data (C) represent the means of the independent experiments which are repeated three times $\left({ }^{*} \mathrm{P}<0.05\right.$ compared with $\left.0 \mathrm{~h}\right)$. (B) After being pre-treated with $\mathrm{SMA}(0.1 \mu \mathrm{M})$ for $4 \mathrm{~h}$ or transient transfected with PKC $\varepsilon$ plasmid for $24 \mathrm{~h}$, BECs were lysed. In groups for scratching, cells were pre-treated with GF109203X (20 $\mu \mathrm{M}$ ) or not (control) for $3 \mathrm{~h}$, then scratched and incubated for $1 \mathrm{~h}$. Cells were lysed before (-) or $1 \mathrm{~h}$ after (+) scratching. Phosphorylated-serine was detected in the equal amounts of precipitates. Data (D) represent the means from three independent experiments $\left({ }^{*} \mathrm{P}<0.05\right.$ compared with the control before scratching). (E) BECs were lysed before (-) or $1 \mathrm{~h}$ after (+) scratching. IQGAP1 and PKC $\varepsilon$ were immunoprecipitated (IP) with each other. The figure represents the independent experiments which are repeated three times. (F) After treatment with PMA $(0.1 \mu \mathrm{M})$, GF109203X (20 $\mu \mathrm{M})$ or transfection of PKC $\varepsilon$ for $24 \mathrm{~h}$, $16 \mathrm{HBE} 14 \mathrm{o}-$ cells were scratched. The closure rates of the wound gap were measured at different time points (Materials and methods). Data (F) represent the means of six independent wells from two separate experiments $\left({ }^{*} \mathrm{P}<0.05\right.$ compared with control).

of PKC $\varepsilon, 16 \mathrm{HBE} 140$ - cells were scratched. The closure rates of the wound gap were measured at different time points as mentioned in Materials and methods. Our data suggested that overexpression of PKC $\varepsilon$ and treatment with PMA promoted wound closure. On the contrary, the group treated with GF109203X was shown to impair the wound closure (Fig. 2F) $\left({ }^{*} \mathrm{P}<0.05\right.$ compared with the control).

IQGAP1 was involved in wound closure and $\beta$-catenin nuclear transposition. Since a previous finding suggests that IQGAP1 is involved in B-catenin redistribution and activation (27), we think further studies are valuable. Overexpression or knockdown of IQGAP1 was used to study its function. The efficiency of interference was evaluated by RT-PCR and Western blotting. IQGAP1 and $\beta$-actin (positive control) were confirmed knocked down in mRNA and protein after interference (Fig. 3A and B). After the depletion of IQGAP1, 16HBE140- cells were scratched and the closure rates of the wound gap were measured at different time points. We found that the wound had already closed within $24 \mathrm{~h}$ in the control (Fig. 1A), while the knock-down of IQGAP1 blocked the wound closure (Fig. 3C and D).
Then, the nuclear and cytoplasmic proteins were isolated in BECs and then subjected to examination of the expression of $\beta$-catenin. We found that most $\beta$-catenin was located in the cytoplasm in the control, while overexpression of IQGAP1 or scratching for $6 \mathrm{~h}$ induced transposition of $\beta$-catenin from the cytoplasm into the nucleus (Fig. 3E). Under the same conditions, the total amounts of $ß$-catenin were greater than the control (Fig. 3F and G). The transposition of $\beta$-catenin and the total amounts of $\beta$-catenin were attenuated by siIQGAP1.

IQGAPI induced activation of Tcf/Lef and expression levels of c-myc and cyclin D1. B-catenin accumulation in the nucleus acts with Tcf/Lef in order to form a transcriptional complex and promotes expression levels of target genes (28). We tried to verify that the overexpression of IQGAP1 activated the Tcf/Lef signal through the nuclear transposition of $\beta$-catenin. After being transfected with the wild-type of IQGAP1, BECs were co-transfected with Tcf luciferase reporter plasmids. The transfection efficiency was viewed by a fluorescent microscope (data not shown) and then confirmed by immunoprecipitation with anti-IQGAP1 and 


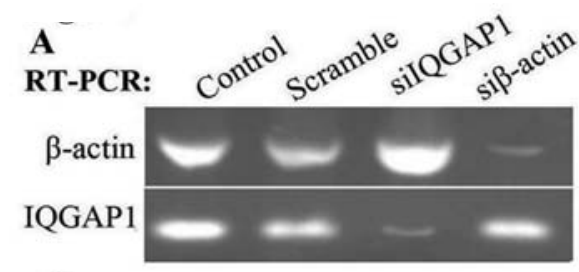

C

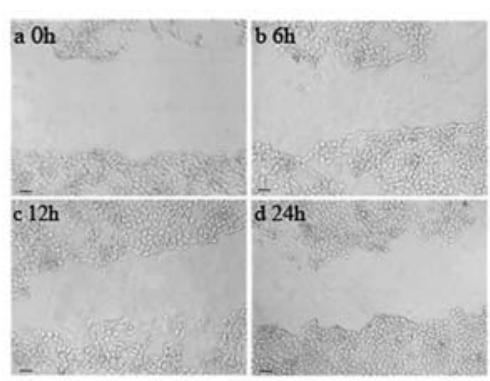

$\mathbf{E}$

WB:
BB:

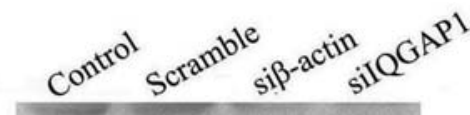

$\beta$-actin

IQGAP1
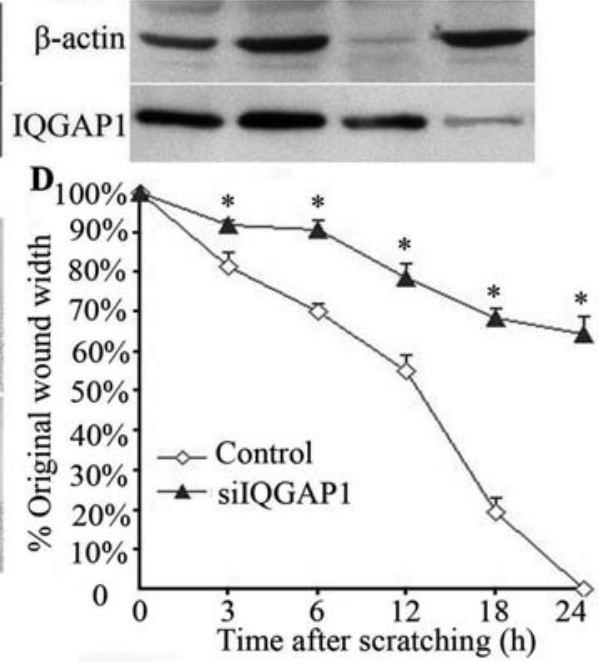

Nuclear
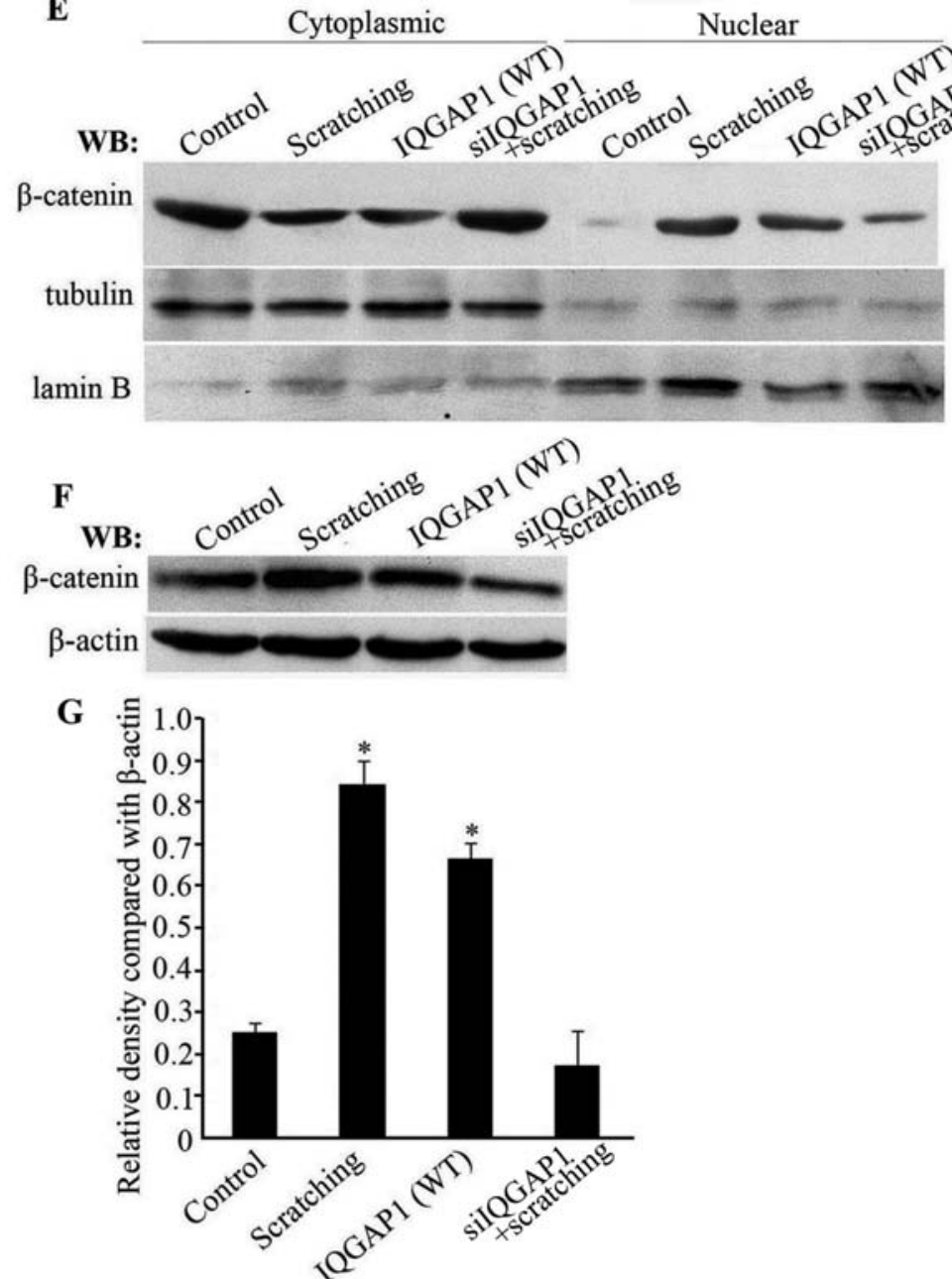

Figure 3. IQGAP1 was involved in wound closure and induced increasing amounts and transposition of ß-catenin. (A) 16HBE14o- cells were transfected with siRNA of IQGAP1 (siIQGAP1, $100 \mathrm{nM}$ ) by LF2000 (2 $\mu \mathrm{l} / \mathrm{well}$ of a 12-well plate), together with siRNA of B-actin (siß-actin, $100 \mathrm{nM})$ and scramble (100 nM) served as a positive and negative control. After interference for $24 \mathrm{~h}$, total mRNA was collected. RT-PCR was carried out and the specific products (IQGAP1, $140 \mathrm{bp}$; B-actin, $564 \mathrm{bp}$ ) were assessed by $2 \%$ agarose gel electrophoresis. The results represent three independent experiments. (B) After being transfected with the above siRNA for $36 \mathrm{~h}, 16 \mathrm{HBE} 14 \mathrm{o}-$ cells were lysed and subjected to SDS-PAGE with anti-IQGAP1 and anti-B-actin antibodies. These figures represent the independent experiments, which are repeated three times. (C) After being transfected with silQGAP1 for $24 \mathrm{~h}, 16 \mathrm{HBE} 14 \mathrm{o}-$ cells were scratched. The closure rates of the wound gap were measured at different time points. Scale bar, $100 \mu \mathrm{m}$. Data (D) represent the means of six independent wells from two separate experiments ( $\mathrm{P}<0.05$ compared with the control). (E) After overexpression and reduction of IQGAP1 or scratched for $6 \mathrm{~h}$, BECs were lysed with NE-PER ${ }^{\mathrm{TM}}$ nuclear and cytoplasmic extraction regents to get cytoplasmic and nuclear extracts according to the instructions from the manufacturer. Then, the supernatant was subjected to SDS-PAGE and immunoblotted (WB) with anti-B-catenin antibody, together with the nuclear marker lamin B and cytoplasmic marker tubulin. This figure shows representative experiments, which are independently repeated three times. (F) Under the above conditions, 16HBE14o- cells were lysed and immunoblotted for total $\beta$-catenin. Data $(\mathrm{G})$ represent the means from three independent experiments ( $\mathrm{P}<0.05$ compared with the control). 


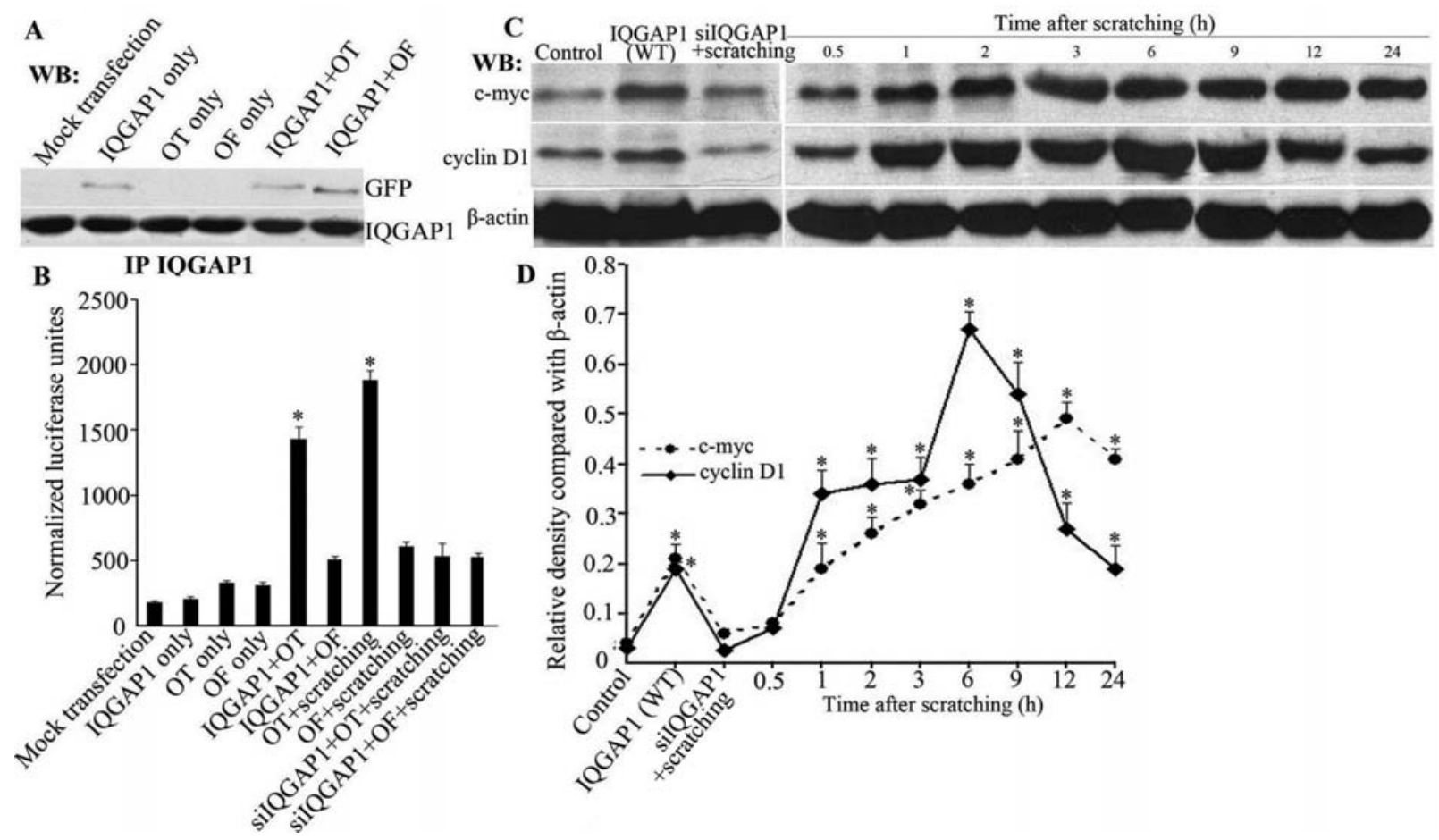

Figure 4. IQGAP1 activated Tcf/Lef and increased the amounts of c-myc and cyclin D1. (A) After being transfected with IQGAP1 and (or) luciferase reporter plasmids, 16HBE14o- cells were lysed and then immunoprecipitated (IP) and immunoblotted with anti-IQGAP1 and anti-GFP antibodies. This result confirmed the efficiency of transient transfection. The figure represents three independent experiments. (B) 16HBE14o- cells were transfected with luciferase reporter plasmids and then treated as described in Materials and methods. OT or OF indicated the groups transfected with pGL3-OT or OF (containing three copies of wild-type or mutant human Tcf-4 binding site). The activities of luciferase and B-galactosidase were detected using the commercial kits according to the manufacturer's instructions and then were normalized for B-galactosidase activity (OD420). Data (B) represent the means from three independent experiments $\left({ }^{*} \mathrm{P}<0.05\right.$ compared with the other control). (C) After the overexpression or reduction of IQGAP1, 16HBE14o- cells were lysed and immunoblotted with anti-c-myc, anti-cyclin D1 and anti-ß-actin antibodies. At the same time, BECs were scratched at different time points and immunoblotted with the above antibodies. Data (D) represent the means of the independent experiments which are repeated three times ( ${ }^{*} \mathrm{P}<0.05$ compared with the control in each group).

anti-GFP antibodies (Fig. 4A). The normalized luciferase activities in groups (pGL3-OT + IQGAP1 and pGL3-OT + scratching) were greater than the other control (Fig. 4B) $\left({ }^{*} \mathrm{P}<0.05\right.$ compared with the other control). Moreover, the activation of Tcf induced by scratching was abolished by siIQGAP1.

C-myc and cyclin D1 have a Tcf/Lef-binding site in the promoter region and are target genes of the $\beta$-catenin/Tcf pathway that promotes cell proliferation $(28,29)$. After overexpression of IQGAP1 in BECs, the amounts of c-myc and cyclin D1 were greater than the control (Fig. 4C). Similar to the expression of IQGAP1, the amounts of c-myc and cyclin D1 also increased and reached a maximum at 12 or $6 \mathrm{~h}$ during the process of wound closure, respectively, though this result was attenuated by the transfection of siIQGAP1 (Fig. 4C and D) $\left({ }^{*} \mathrm{P}<0.05\right.$ compared with the control in each group).

\section{Discussion}

In the present study, we examined the expression and phosphorylation of IQGAP1 in the injury and repair process of BECs induced by scratching. We found that the process of wound closure lasted for $\sim 24 \mathrm{~h}$. After scratching, the amounts of IQGAP1 in mRNA and protein were greater than the control. The phosphorylation of IQGAP1 mediated by PKC $\varepsilon$ was involved in wound closure induced by scratching. The knock-down of IQGAP1 blocked the wound closure, attenuated B-catenin transposition, inactivated Tcf/Lef and suppressed expression levels of its target genes of c-myc and cyclin D1. Our results suggest that IQGAP1 mediated cell proliferation and the phosphorylation of IQGAP1 was involved in the wound closure of BECs.

As IQGAP1 is associated with targets of cell cytoskeleton and adhesion, it is significant to know whether or how the IQGAP1 signal works during the process of injury and repair. We found that scratching increased the amounts of IQGAP1 in mRNA and protein. Their maximum levels were shown at the middle stage of the process. These results indicated that the increasing amounts of IQGAP1 should play role in the process of wound closure. At $6 \mathrm{~h}$ after scratching, B-catenin was transmitted from extranuclear to intranuclear. More importantly, the enforced overexpression of wild-type IQGAP1 promoted $\beta$-catenin nuclear transposition. Under the same conditions, the amounts of total $\beta$-catenin increased. These data suggested a new pathway that IQGAP1 was involved in increasing amounts of and transposition of $ß$-catenin in wound closure.

Through the activation of Tcf signaling, $\beta$-catenin plays an important role in normal cutaneous wound repair (30). We then sought to study the function of B-catenin transposition induced by the overexpression of IQGAP1 in BECs. The Tcf luciferase reporter system was used to test the activation signal of Tcf. The wild-type or mutant of the human Tcf-4 binding site has been cloned into pGL3-OT or OF, respectively. When Tcf binds the site in pGL3-OT, the luciferase gene in the 
downstream is activated. Our previous results gave direct evidence that the nuclear transposition of $\beta$-catenin and Tcf activation are involved in the wound closure induced by scratching (19). In this experiment, we found that enforced overexpression of IQGAP1 activated the Tcf signal. The expression levels of c-myc and cyclin D1 are dependent on the $\beta$-catenin/Tcf signal and have a direct effect on cell proliferation $(29,31,32)$. After scratching or overexpression of IQGAP1, the amounts of c-myc and cyclin D1 increased, which were analogous to the expression of IQGAP1. At the same time, the suppression of IQGAP1 blocked scratchinginduced wound closure, B-catenin transposition and Tcf activation. All these data supported our predictions that scratching increased the amounts of IQGAP1, which promoted B-catenin accumulation and nuclear transposition. These effects activated the Tcf signal and mediated the proliferation in the injured BECs.

A novel type of phosphorylated IQGAP1 is revealed, which modulates the interaction with the cytoskeleton and promotes neurite outgrowth $(15,16)$. These findings guide us to study whether IQGAP1 is analogously phosphorylated in the process of injury and repair. Since the serine residues of IQGAP1 were easily phosphorylated (15), we examined the phosphorylated serine instead of phosphorylation of IQGAP1. Soon after scratching the BECs, the amounts of the phosphorylated serines increased and reached a maximum at $1 \mathrm{~h}$, lasting for $\sim 9 \mathrm{~h}$. These results were not caused by the increasing amounts of IQGAP1. The maximum of IQGAP1 in protein was observed at $9 \mathrm{~h}$ after scratching, which was later than that of the phosphorylated serine $(1 \mathrm{~h})$. Furthermore, the phosphorylation of IQGAP1 was detected in equal amounts of precipitated proteins in our coimmunoprecipitation experiment.

PKC $\varepsilon$ is suggested to be a candidate for the phosphorylation of IQGAP1 (15) and small GTPase signal (33). The latter mediates morphological and cytoskeletal effects in neural cells and fibroblasts. We further found that enforced overexpression of PKC $\varepsilon$ and treatment with PMA increased the amounts of phosphorylation of IQGAP1, while treatment with GF109203X blocked the phosphorylation of IQGAP1 induced by scratching. IQGAP1 and PKC $\varepsilon$ were confirmed to interact with each other, which was affected by scratching. Moreover, direct evidence was given that phosphorylation of IQGAP1 was involved in wound closure after treatment with PMA, GF109203X or overexpression of $\mathrm{PKC} \varepsilon$ in BECs. These results suggested that $\mathrm{PKC} \varepsilon$ mediated the phosphorylation of IQGAP1, which was involved in the early stages of wound closure.

Taken together, we conclude that wound closure of the airway epithelial cells involves several coordinated events that are dependent on the upregulation and phosphorylation of IQGAP1. Phosphorylation of IQGAP1 occurs at the early stage of injury and repair, while increasing amounts of IQGAP1 appears at the middle stage. These results activate Tcf and promote cell proliferation. It is an important step that the airway epithelium initiates the wound repair process after injury in the resolution of airway diseases such as chronic bronchitis and COPD, in which the basal cells of the airway proliferate and differentiate into a ciliated epithelium to recovery of the injured epithelium $(3,34)$. Our results of IQGAP1 and the phosphorylation of IQGAP1 signals involved in the airway epithelial cells may be helpful for exploring the mechanisms of these diseases. Further study will concentrate on the function of IQGAP1 on the cytoskeleton and the regulation of phosphorylation of IQGAP1 in wound closure.

\section{Acknowledgements}

We are grateful to Professor Kozo Kaibuchi for the plasmid of pEGFP-IQGAP1 (WT), to Dr B. Vogelstein for the Tcf luciferase reporter plasmids, to Professor Cao Xi'nan for pCDNA3-PKC $\varepsilon$ plasmid and to Dr D. Gruenert for the 16HBE14o- cells. We also thank Professor Wang Xi and Dr Chen Wenshu for reviewing this manuscript and providing insightful suggestions. This study was supported by a grant from the National Natural Science Foundation of P.R. China (No. 30470757).

\section{References}

1. Farooqui R and Fenteany G: Multiple rows of cells behind an epithelial wound edge extend cryptic lamellipodia to collectively drive cell-sheet movement. J Cell Sci 118: 51-63, 2005.

2. Adam EC, Holgate ST, Fildew CJ and Lackie PM: Role of carbohydrates in repair of human respiratory epithelium using an in vitro model. Clin Exp Allergy 33: 1398-1404, 2003.

3. Sacco O, Silvestri M, Sabatini F, Sale R, Defilippi AC and Rossi GA: Epithelial cells and fibroblasts: structural repair and remodeling in the airways. Paediatr Respir Rev 5: S35-S40, 2004.

4. Burridge $\mathrm{K}$ and Wennerberg $\mathrm{K}$ : Rho and Rac take center stage. Cell 116: 167-179, 2004

5. Bashour A, Fullerton AT, Hart MJ and Bloom GS: IQGAP1, a Rac- and cdc42- binding protein, directly binds and cross-links microfilaments. J Cell Biol 137: 1555-1566, 1997.

6. Fukata M, Kuroda S, Nakagawa M, et al: Cdc42 and Rac1 regulate the interaction of IQGAP1 with B-catenin. J Biol Chem 274: 26044-26050, 1999.

7. Kuroda S, Fukata M, Nakagawa M, et al: Role of IQGAP1, a target of the small GTPases Cdc42 and Rac1, in regulation of Ecadherin-mediated cell-cell adhesion. Science 281: 832-835, 1998.

8. Fukata M, Watanabe T, Noritake J, et al: Rac1 and Cdc42 capture microtubules through IQGAP1 and CLIP-170. Cell 109: 873-885, 2002.

9. Watanabe T, Wang S, Noritake J, et al: Interaction with IQGAP1 links APC to Rac1, Cde42 and actin filaments during cell polarization and migration. Dev Cell 7: 871-883, 2004.

10. Kholmanskikh SS, Koeller HB, Wynshaw-Boris A, Gomez T, Letourneau PC and Ross ME: Calcium-dependent interaction of Lis1 with IQGAP1 and cdc42 promotes neuronal motility. Nat Neurosci 9: 50-57, 2006.

11. Noritake J, Watanabe T, Sato K, Wang S and Kaibuchi K: IQGAP1: a key regulator of adhesion and migration. J Cell Sci 118: 2085-2092, 2005.

12. Brown MD and Sacks DB: IQGAP1 in cellular signaling: bridging the GAP. Trends Cell Biol 16: 242-249, 2006.

13. Loureiro JJ, Rubinson DA, Bear JE, Baltus GA, Kwiatkowski AV and Gertler FB: Critical roles of phosphorylation and actin binding motifs, but not the central proline-rich region, for Ena/ vasodilator-stimulated phosphoprotein (VASP) function during cell migration. Mol Biol Cell 13: 2533-2546, 2002.

14. Chikumi H, Fukuhara S and Gutkind JS: Regulation of G protein-linked guanine nucleotide exchange factors for Rho, PDZ-RhoGEF, and LARG by tyrosine phosphorylationevidence of a role for focal adhesion kinase. J Biol Chem 277: 12463-12473, 2002.

15. Grohmanova K, Schlaepfer D, Hess D, Gutierrez P, Beck M and Kroschewski R: Phosphorylation of IQGAP1 modulates its binding to $\mathrm{Cdc} 42$, revealing a new type of rho-GTPase regulator. J Biol Chem 279: 48495-48504, 2004.

16. Li Z, McNulty DE, Marler KJ, Lim L, Hall C, Annan RS and Sacks DB: IQGAP1 promotes neurite outgrowth in a phosphorylation-dependent manner. J Biol Chem 280: 13871-13878, 2005. 
17. Tian D, Zhu M, Chen WS, Li JS, Wu RL and Wang X: Role of glycogen synthase kinase 3 in squamous differentiation induced by cigarette smoke in porcine tracheobronchial epithelial cells. Food Chem Toxicol 44: 1590-1596, 2006.

18. Zhu M, Li J, Tian D, Ma Y, Li N and Wu R: Spatial-temporal distribution of glycogen synthase kinase $3 \beta$ and adenomatous ployposis coli protein are involved in the injury and repair of airway epithelial cells induced by scratching. Acta Physiol Sinica 59: 197-203, 2007.

19. Zhu M, Tian D, Li J, Ma Y, Wang Y and Wu R: Glycogen synthase kinase $3 \beta$ and $\beta$-catenin are involved in the injury and repair of bronchial epithelial cells induced by scratching. Exp Mol Pathol 83: 30-38, 2007

20. Ma Y, Li N, Wu R, Liu M, Hong Y, Zhu M and Tian D: Dynamic changes of cytoplasmic linker protein-170 in the process of airway epithelial injury and repair in mice. Acta Med Univ Sci Technol Huazhong 36: 1-4, 2007.

21. Etienne-Manneville S and Hall A: Cdc42 regulates GSK-3ß and adenomatous polyposis coli to control cell polarity. Nature 421: 753-756, 2003.

22. Cozens AL, Yezzi MJ, Kunzelmann K, et al: CFTR expression and chloride secretion in polarized immortal human bronchial epithelial cells. Am J Respir Cell Mol Biol 10: 38-47, 1994.

23. Livak KJ and Schmittgen TD: Analysis of relative gene expression data using real-time quantitative PCR and the 2(-Delta Delta CT) method. Methods 25: 402-408, 2001.

24. Fukata M, Nakagawa M, Itoh N, Kawajiri A, Yamaga M, kuroda S and Kaibuchi K: Involvement of IQGAP1, an effector of Rac1 and Cdc42 GTPases, in cell-cell dissociation during cell scattering. Mol Cell Biol 21: 2165-2183, 2001.

25. Korinek V, Barker N, Morin PJ, et al: Constitutive transcriptional activation by a $\beta$-catenin-Tcf complex in $\mathrm{APC}^{-}-$- colon carcinoma. Science 275: 1784-1787, 1997.
26. Tucker TA, Varga K, Bebok Z, et al: Transient transfection of polarized epithelial monolayers with CFTR and reporter genes using efficacious lipid. Am J Physiol Cell Physiol 284: C791-C804, 2003.

27. Briggs MW, Li Z and Sacks DB: IQGAP1-mediated stimulation of transcriptional co-activation by $\beta$-catenin is modulated by calmodulin. J Biol Chem 277: 7453-7465, 2002.

28. Cheon SS, Nadesan P, Poon R and Alman BA: Growth factors regulate beta-catenin-mediated TCF-dependent transcriptional activation in fibroblasts during the proliferative phase of wound healing. Exp Cell Res 293: 267-274, 2004.

29. Tetsu O and Mccormick F: Beta-catenin regulates expression of cyclin D1 in colon carcinoma cells. Nature 398: 422-426, 1999.

30. Cheon SS, Wei Q, Gurung A, et al: Beta-catenin regulates wound size and mediates the effect of TGF-beta in cutaneous healing. FASEB J 20: 692-701, 2006.

31. He TC, Sparks AB, Rago C, et al: Identification of c-myc as a target of the APC pathway. Science 281: 1509-1512, 1998

32. Natsume H, Sasaki S, Kitagawa M, et al: B-Catenin/Tcf-1mediated transactivation of cyclin D1 promoter is negatively regulated by thyroid hormone. Biochem Biophys Res Commun 309: 408-413, 2003.

33. Trollér U and Larsson C: $\mathrm{Cdc} 42$ is involved in $\mathrm{PKC} \varepsilon-$ and $\delta$ induced neurite outgrowth and stress fibre dismantling. Biochem Biophys Res Commun 349: 91-98, 2006.

34. Spurzem JR, Veys T, Devasure J, Sisson JH and Wyatt TA: Ethanol treatment reduces bovine bronchial epithelial cell migration. Alcohol Clin Exp Res 29: 485-492, 2005. 